\title{
BMJ Open Examining the effects of low back pain and mental health symptoms on healthcare utilisation and costs: a protocol for a population-based cohort study
}

Jessica J Wong, ${ }^{1,2}$ Pierre Côté, ${ }^{1,2,3,4}$ Andrea C Tricco, ${ }^{1,4,5}$ Laura C Rosella (iD) ${ }^{1,6}$

To cite: Wong JJ, Côté P, Tricco AC, et al. Examining the effects of low back pain and mental health symptoms on healthcare utilisation and costs: a protocol for a populationbased cohort study. BMJ Open 2019;9:e031749. doi:10.1136/ bmjopen-2019-031749

- Prepublication history for this paper is available online. To view these files, please visit the journal online (http://dx.doi. org/10.1136/bmjopen-2019031749).

Received 16 May 2019 Revised 09 August 2019 Accepted 02 September 2019

Check for updates

(C) Author(s) (or their employer(s)) 2019. Re-use permitted under CC BY-NC. No commercial re-use. See rights and permissions. Published by BMJ.

For numbered affiliations see end of article.

Correspondence to

Dr Laura C Rosella;

laura.rosella@utoronto.ca

\section{ABSTRACT}

Introduction Low back pain (LBP) is a leading cause of disability associated with high healthcare utilisation and costs. Mental health symptoms are negative prognostic factors for LBP recovery; however, no population-based studies have assessed the joint effects of LBP and mental health symptoms on healthcare utilisation. This proposed study will characterise the health system burden of LBP and help identify priority groups to inform resource allocation and public health strategies. Among community-dwelling adult respondents of five cycles of the Canadian Community Health Survey (CCHS) in Ontario, we aim to assess the effect of self-reported LBP on healthcare utilisation and costs and assess whether this effect differs between those with and without self-reported mental health symptoms.

Methods and analysis We designed a dynamic population-based cohort study using linkages of survey and administrative data housed at ICES. The Ontario sample of CCHS (2003-2004, 2005-2006, 2007/2008, 2009/2010, 2011/2012; total of $\sim 130000$ eligible respondents) will be used to define the cohort of adults with self-reported LBP with and without mental health symptoms. Healthcare utilisation and costs will be assessed by linking health administrative databases. Follow-up ranges from 6 to 15 years (until 31 March 2018). Sociodemographic (eg, age, sex, education) and health behaviour (eg, comorbidities, physical activity) factors will be considered as potential confounders. Poisson and linear (log-transformed) regression models will be used to assess the association between LBP and healthcare utilisation and costs. We will assess effect modification with mental health symptoms on the additive and multiplicative scales and conduct sensitivity analyses to assess the impact of misclassification and residual confounding.

Ethics and dissemination This study is approved by the University of Toronto Research Ethics Board. We will disseminate findings using a multifaceted knowledge translation strategy, including scientific conference presentations, publications in peer-reviewed journals and workshops with key knowledge users.

\section{INTRODUCTION}

The WHO recognises disability as a global public health issue and priority area for
Strengths and limitations of this study

- This will be the first population-based cohort study to assess the joint effects of low back pain (LBP) and mental health symptoms on healthcare utilisation and costs.

- We will consider and control for a wide range of sociodemographic and health behaviour factors as potential confounders of the association between LBP and healthcare utilisation/costs.

- Although we will use both self-reported LBP and LBP diagnostic information to ascertain the exposure, misclassification may occur; however, this approach currently represents the best secondary data methods to capture LBP in the general population.

- Our primary analysis will exclude services not covered by the Ontario provincial health insurance system (eg, physiotherapy, chiropractic care), which may underestimate the utilisation of healthcare services for LBP.

- Residual confounding may bias our results, but we will conduct a quantitative bias analysis to estimate the extent to which residual confounding may explain some or all of the reported association between LBP and healthcare utilisation/costs.

action. ${ }^{1}$ Low back pain (LBP) is the leading cause of years lived with disability globally and can be related to occupational injuries or motor vehicle collisions. ${ }^{2-4}$ It is a common condition, as approximately $80 \%$ of people experience at least one episode of LBP during their lives. ${ }^{25}$ Although most episodes resolve, a considerable proportion $(\sim 10 \%-20 \%)$ of adults with LBP experience chronic symptoms, functional limitations or difficulties returning to work. ${ }^{67}$

LBP is burdensome to patients and health systems, as it is associated with high healthcare use and costs. ${ }^{8-10}$ In the USA, estimated healthcare spending for LBP and neck pain was US $\$ 87.6$ billion in 2013 , which was the 
third highest after diabetes and ischaemic heart disease. ${ }^{11}$ Moreover, healthcare spending for LBP and neck pain increased US $\$ 57.2$ billion over 18 years, the second highest increase after diabetes. ${ }^{11}$ Among musculoskeletal conditions, LBP is the most common reason for seeking healthcare. ${ }^{1213}$ Health Quality Ontario published a quality standard in 2019 with aims to ensure that all Ontarians receive the same high-quality care for LBP. ${ }^{14}$ This quality standard provides guidance on reducing unnecessary diagnostic imaging, encouraging physical activity, providing education and reassurance and assisting with self-management and treatment options for adults with acute LBP. ${ }^{14}$ Health Quality Ontario published this quality standard in response to the identified need to improve LBP care in Ontario. ${ }^{14}$ In Ontario, costs for spinal imaging have increased 55\% from 2001/2002 to 2010/2011 (increases greater than any other Canadian health service) with considerable geographical variation in access to appropriate LBP care. ${ }^{14}$

High healthcare utilisation and costs are likely driven by high-risk subgroups with LBP. ${ }^{15}$ In the USA, two-thirds of patients with LBP had 1-2 encounters (ie, encounters in primary care, specialty care or emergency department (ED), surgery, drug prescription, lab or imaging orders) for LBP over 5 years, while approximately $10 \%$ of patients had heavy (eg, accounting for $>50 \%$ of all LBP services) utilisation related to imaging, injections, ED visits or inpatient encounters. ${ }^{15}$ LBP subgroups with greater healthcare utilisation tend to have more comorbidities (eg, cardiovascular diseases, diabetes) and symptoms of depression or anxiety. ${ }^{15}$ Health programmes could target high-risk groups to reduce the burden of chronic conditions, but these priority groups for LBP have not been well defined.

Comorbid mental health symptoms may predispose individuals to be at higher risk of high healthcare utilisation. Mental health symptoms are strongly linked with LBP, and research that further assesses their joint effects on healthcare utilisation is needed. Evidence suggests that mental health symptoms are negative prognostic factors for LBP. ${ }^{16-21}$ People with mental health symptoms, such as depressive symptoms or anxiety, have poorer recovery from LBP. ${ }^{16-19}$ Preliminary evidence suggests that symptoms of depression or anxiety are more common in patients with LBP who more heavily use care. ${ }^{15}$ However, some studies were cross-sectional, while previous cohort studies relied on relatively short follow-up $(<3$ years) or did not adequately control for a wide range of confounders (eg, comorbidities, health-related/behaviour factors). ${ }^{15}$ 22-25 To date, no population-based cohort study has assessed the joint effects of LBP and mental health symptoms on healthcare utilisation and costs.

Assessing the joint effects of LBP and mental health symptoms is needed to inform resources and health services delivery planning for priority subgroups with LBP. From a clinical perspective, mental health symptoms are common comorbidities presenting in patients with LBP. $^{26-29}$ Studying the joint effects between LBP and mental health symptoms will allow us to determine the burden of these two prevalent conditions on healthcare utilisation and costs to better inform healthcare programmes in Canada.

\section{Objectives}

Our goal is to examine the effect of LBP and the joint effects of LBP and mental health symptoms on future healthcare utilisation and costs among adults in Ontario, using linked survey and healthcare utilisation data. Specifically, we aim to achieve the following objectives.

Among five cycles of Ontario respondents of the Canadian Community Health Survey (CCHS) who are community-dwelling adults aged $\geq 18$ years:

- Objective 1: To assess the effect of self-reported LBP (compared with no self-reported LBP) on healthcare utilisation and costs.

- Objective 2: To assess whether the effect of self-reported LBP on healthcare utilisation and costs differs for those with self-reported mental health symptoms compared with those who do not report mental health symptoms.

\section{METHODS AND ANALYSIS}

\section{Study design}

We will conduct a dynamic population-based cohort study of Ontario adult respondents of the CCHS. Ontario is the largest province by population ( 14.2 million in 2017) in Canada, and the most ethnically diverse province with more than 200 ethnic origins represented.$^{30}$ In Ontario, many healthcare services, including visits to family physicians and specialists, and most basic and emergency healthcare services (eg, surgery and hospital stays) are publicly funded. ${ }^{31}$ These services are paid through the government-run provincial health insurance plan (Ontario Health Insurance Plan (OHIP)).

\section{Study sample}

All Ontario respondents from five CCHS cycles (Cycle 2.1 (2003-2004), Cycle 3.1 (2005-2006), 2007/2008, $2009 / 2010,2011 / 2012$ ) aged $\geq 18$ years at time of survey will form the cohort. Before 2007, CCHS data were collected from a sample of respondents every 2 years (ie, 2003-2004, 2005-2006). Since 2007, data are collected from a sample of respondents yearly, and a file combining 2 years of data is released (ie, 2007/2008, 2009/2010, 2011/2012). ${ }^{32}$ We will exclude individuals who cannot be linked with health administrative databases. Approximately 130000 Ontario respondents to the CCHS are eligible for this study.

\section{Data sources}

In addition to providing LBP information, CCHS survey data will provide mental health symptoms, socioeconomic status and health behaviour information. Details of data elements from each data sources can be found in table 1 and are listed here.

- CCHS (annual component on general health): CCHS is administered by Statistics Canada and gathers 
Table 1 Data elements and data sources

\begin{tabular}{|c|c|c|}
\hline Data group & Data element & Data source \\
\hline Exposure & Self-reported low back pain & $\mathrm{CCHS}$ \\
\hline \multirow{2}{*}{$\begin{array}{l}\text { Effect measure } \\
\text { modifier }\end{array}$} & Poor self-perceived mental health & $\mathrm{CCHS}$ \\
\hline & Anxiety disorder & $\mathrm{CCHS}$ \\
\hline \multirow[t]{7}{*}{ Potential confounders } & Age & RPDB \\
\hline & Educational attainment & $\mathrm{CCHS}$ \\
\hline & Disability & OSAD \\
\hline & Smoking & $\mathrm{CCHS}$ \\
\hline & Alcohol consumption & $\mathrm{CCHS}$ \\
\hline & Physical activity & $\mathrm{CCHS}$ \\
\hline & Comorbidities & OHIP, ClHI \\
\hline \multirow[t]{5}{*}{ Outcome measures } & Number of physician visits & OHIP \\
\hline & Number of ED visits & NACRS \\
\hline & Number of hospitalisations and stay days & CIHI Discharge Abstract Database/Same Day Surgeries \\
\hline & Spinal imaging procedures & $\mathrm{OHIP}$ and $\mathrm{CIHI}$ \\
\hline & Healthcare costs & $\begin{array}{l}\text { ICES costing methodology using: } \\
\text { RPDB: demographic information, date of death for the } \\
\text { entire population with valid health card numbers } \\
\text { Discharge Abstract Database: acute inpatient } \\
\text { hospitalisation } \\
\text { Ontario Mental Health Reporting System: mental } \\
\text { health } \\
\text { - Continuing Care Reporting System: complex } \\
\text { continuing care } \\
\text { National Rehabilitation System: inpatient rehabilitation } \\
\text { National Ambulatory Care Reporting System: same } \\
\text { day surgeries and emergency department, outpatient } \\
\text { oncology and dialysis treatments } \\
\text { OHIP: physician services } \\
\text { Ontario Drug Benefit and New Drug Funding Program: } \\
\text { prescription drugs } \\
\text { Ontario Home Care Administrative System and Home } \\
\text { Care Database: home care } \\
\text { Assistive Devices Program database: assistive } \\
\text { devices }\end{array}$ \\
\hline
\end{tabular}

CCHS, Canadian Community Health Survey; CIHI, Canadian Institute for Health Information; NACRS, National Ambulatory Care and Reporting System; OHIP, Ontario Health Insurance Plan; OSAD, Ontario Social Assistance Database; RPDB, Registered Persons Database.

up-to-date, cross-sectional data on the distribution of health determinants, outcomes and healthcare use across Canada. ${ }^{32}$ CCHS is a cross-sectional survey of the Canadian population that uses a multistage survey design. To provide reliable estimates, CCHS aimed for a sample of 130000 respondents every 2 years before 2007 and a sample of 65000 respondents annually after $2007 .^{32}$ Survey weights are available for calculations in order for estimates produced from survey data to be representative of the covered population. ${ }^{32}$ CCHS data are representative of $98 \%$ of the Canadian population aged $\geq 12$ years living in private dwellings at national and provincial levels with response rates $>75 \% .{ }^{32}$ More than $80 \%$ of CCHS respondents have agreed to and have valid health card numbers for linkage to Registered Persons Database (RPDB) data. Survey methodology is detailed elsewhere. ${ }^{33}$

- CCHS (focus content surveys on mental health): We will use 2002 (Cycle 1.2) and 2012 CCHS focus content surveys examining mental health. The content is partly based on select mental disorders from the World Mental Health-Composite International Diagnostic Interview 
Instrument, measured according to Diagnostic and Statistical Manual of Mental Disorders, fourth edition definitions. ${ }^{3435}$

- OHIP: OHIP covers all Ontario residents, including all survey respondents, as a single-payer health insurance system. These data cover all healthcare providers who can claim OHIP (eg, physicians, laboratories) and include service codes, dates of service and associated diagnosis. ${ }^{36}$ We will use OHIP physician claims file to ascertain ambulatory physician visits and imaging (radiographs, CT, MRI).

- Canadian Institute for Health Information (CIHI): CIHI Discharge Abstract Database and Same Day Surgeries collect demographic, administrative and clinical data on hospital discharges and same day surgeries, which are received from acute care facilities, health/ regional authority or ministry of health, depending on the province. We will use this to obtain data on hospitalisations and imaging.

- National Ambulatory Care Reporting System (NACRS): This captures data on all hospital-based and community-based ambulatory care, collected from specific facilities, regional health authorities and ministries of health. This will be used to ascertain data on ED visits and imaging.

- RPDB: The RPDB captures demographic information on all individuals who received a health card number in Ontario and updates addresses when an OHIP card is issued or renewed, or with address changes registered in EDs or hospitals. This will be used to identify demographic information, and censoring when subjects move out of Ontario.

- Social Assistance Data-Ministry of Community and Social Services: We will use administrative caseload data provided by the Ontario Ministry of Community and Social Services, linked at ICES, to identify all users of Ontario's two major social assistance programmes (Ontario Disability Support Program, Ontario Works). ${ }^{37} 38$ It will be used for data on disability and social assistance use (eg, benefit unit pay details, income and deductions skills and training) as covariate information.

Data sources for healthcare costing methodology related to utilisation and cost information are listed in table 1 (detailed information is described elsewhere). ${ }^{39}$

\section{Data linkages}

Data pooled from CCHS will be individually linked to individual-level healthcare utilisation data from administrative databases. Each participant will be linked deterministically and individually to population-based health administrative databases for Ontario. Deterministic linkage is an all-or-nothing linkage approach that is superior to probabilistic linkage, as records are matched using an exact match of unique identifying information. ${ }^{40}$ Specifically, deterministic linkage between CCHS and individual-level health administrative data will be achieved through ICES, using unique healthcare identifiers (encrypted OHIP numbers) to ascertain healthcare utilisation and costs. We will use only data from the first CCHS interview in individuals who partake in multiple survey cycles; however, we anticipate this to be a very small proportion of the entire sample $(<0.5 \%)$.

\section{Definition of exposure}

Self-reported LBP and diagnostic information from healthcare encounters

Self-reported LBP will be obtained from the CCHS question: "Do you have back problems, excluding fibromyalgia and arthritis?" Individuals who respond no to this question will be classified as having no LBP. This CCHS question refers to back problems that are expected to last or have already lasted 6 months and that have been diagnosed by a health professional. Symptoms at least 6 months in duration are considered chronic. ${ }^{41}$ This definition of self-reported LBP has been used in previous studies $^{27}{ }^{42-45}$; however, it has not been tested for its validity and reliability. To address this, we will compare the responses of this information with health administrative data, including billing data that contains diagnostic codes for those with LBP (ie, seeking healthcare for LBP). We will conduct sensitivity analyses to assess the effects for those who self-report LBP with and without prior diagnostic billing information as a more specific definition of LBP among those who seek care (table 2).

\section{Outcomes}

Outcomes of interest are spine-related healthcare utilisation and costs based on diagnostic/procedural codes listed in table 2, as measured by:

- Number of physician visits: Following CCHS interview date, all OHIP documented physician visits outside of hospital settings (fee for service or shadow billed) will be designated as the number of physician visits for each subject.

- Number of ED visits: Following CCHS interview date, all NACRS ED visits will be designated as the number of ED visits for each subject.

- Number of hospitalisations: Following the CCHS interview date, all inpatient hospitalisations and same day surgeries in CIHI will be designated as the number of hospitalisations for each subject.

- Number of spinal imaging procedures: After the CCHS interview date, specific OHIP and CIHI fee codes will identify the number of spine imaging for radiographs, CT and MRI. Previous studies at ICES used these spinal imaging codes related to LBP (table 2). ${ }^{46}$

- Healthcare costs: Total healthcare spending in Canadian dollars (adjusted to 2014) will be calculated using a person-centred costing approach to linked health administrative databases. ${ }^{39}$ This methodology uses an algorithm to estimate costs accrued by each person based on healthcare visits covered by the Ministry of Health and Long Term Care (following CCHS interview date). From 2003 onwards, comprehensive healthcare costs are available for all major sectors 
Table 2 Select diagnostic and procedural codes for (A) spinal imaging (B) low back pain and (C) mental health symptoms

\begin{tabular}{|c|c|}
\hline Condition & Diagnostic or procedural codes \\
\hline \multicolumn{2}{|l|}{ (A) Spinal imaging } \\
\hline Radiographs & X025, X202, X203, X027, X204, X028, X205, X206, X032, X033, X031, X034, X207, X035, X208 \\
\hline СT & $\mathrm{X} 415, \mathrm{X} 416, \mathrm{X} 128$ \\
\hline MRI & X490, X492, X493, X495, X496, X498 \\
\hline (B) Low back pain & $\begin{array}{l}\text { ICD-9: } 722.9,724,749.9,846,847.1 \\
\text { ICD-10: M54.5, M54.1, M54.3, M54.4, M54.8, M54.9 }\end{array}$ \\
\hline \multicolumn{2}{|l|}{ (C) Mental health symptom* } \\
\hline Any mental health disorder & $\begin{array}{l}\text { ICD-9: Any OMHRS (including missing, except for 290.x, 294.x in primary diagnosis). } \\
\text { Exclude if primary dx missing and provisional=2 } \\
\text { ICD-10: DX10CODE1=F06 F99 or DX10CODE2-DX10CODE10=X60-X84, Y10-Y19, Y28 } \\
\text { when DX10CODE1 not equal to F06-F99 }\end{array}$ \\
\hline Substance-related disorders & $\begin{array}{l}\text { ICD-9: 291.x (all } 291 \text { codes, excluding 291.82), } 292 . x \text { (all } 292 \text { codes, excluding 292.85), } \\
\text { 303.x (all 303 codes), 304.x (all } 304 \text { codes), 305.x (all } 305 \text { codes). Provisional=4 } \\
\text { ICD-10: F55, F10-F19 }\end{array}$ \\
\hline Schizophrenia & $\begin{array}{l}\text { ICD-9: } 295 . x \text { (all } 295 \text { codes), 297.x (all } 297 \text { codes), 298.x (all } 298 \text { codes). Provisional=5 } \\
\text { ICD-10: F20 (excluding F20.4), F22, F23, F24, F25, F28, F29, F53.1 }\end{array}$ \\
\hline Mood disorders & $\begin{array}{l}\text { ICD-9: 296.x (all } 296 \text { codes), 300.4x, 301.13, 311.x. Provisional=6 } \\
\text { ICD-10: F30, F31, F32, F33, F34, F38, F39, F53.0 }\end{array}$ \\
\hline Anxiety disorders & $\begin{array}{l}\text { ICD-9: 300, 300.0x, 300.2x, 300.3x, 308.3x, 309.0x, 309.24, 309.28, 309.3x, 309.4x, } \\
\text { 309.8x, 309.9x. Provisional=7, 15 } \\
\text { ICD-10: F40, F41, F42, F43, F48.8, F48.9, F93.1, F93.2 }\end{array}$ \\
\hline
\end{tabular}

${ }^{*}$ For healthcare utilisation specific to mental health symptoms (eg, emergency department visits).

ICD, International Classification of Diseases; NACRS, National Ambulatory Care Reporting System.

of healthcare spending: inpatient hospitalisations, physician visits, complex continuing care, long-term care, home services, assistive devices and pharmaceuticals. ${ }^{39}$ We have successfully applied these methods to estimate attributable costs for other conditions. ${ }^{39} 48-50$

Healthcare utilisation and costs data will be collected until 31 March 2018 (range 6-15 years of follow-up). We will calculate person-time from CCHS interview date to end of study period (31 March 2018), OHIP ineligibility or date of death.

\section{Definitions of covariates/potential confounders}

All potential confounders of the association between LBP and healthcare utilisation/costs will be accounted for in the analysis, using propensity methods for objective 1 and as covariates in models for objective 2 (described in the analysis section). These variables were selected based on previous literature ${ }^{17235152}$ :

- Demographic factors: Age (years), sex (male or female).

- Socioeconomic factors: Household income (lowest to highest quintiles), educational attainment (less than secondary, secondary graduate, more than secondary), occupation group (eg, management, health, sales and service occupations); disability (data from Ontario Social Assistance Database).

- Health-related/behaviour factors:

- Self-reported factors (CCHS): Smoking (heavy, light, former heavy, former light, non-smoker), alcohol consumption (heavy, moderate, light drinker), physical activity quartiles (bottom $25 \%$ to top $25 \%$ physically active), body mass index (normal/underweight, overweight, obese), self-rated general health (excellent/very good/good, fair, poor), self-reported chronic conditions (yes, no).

- Comorbidities using a 2-year look-back window from CCHS interview date (ie, up to 2 years prior to interview date): Aggregated Diagnosis Groups (validated among adults in Ontario) ${ }^{53}$; health conditions using health administrative database algorithms (eg, diabetes, hypertension, congestive heart failure, chronic obstructive pulmonary disease, dementia, stroke, coronary artery disease).$^{54-58}$

\section{Definition of effect measure modifier (for objective 2)}

Self-reported mental health symptoms

Effect modifiers of interest are the following mental health symptoms: poor self-perceived mental health, mood disorder and anxiety disorder. We will examine individual and composite measures of these mental health symptoms. We will also conduct subanalyses using data from CCHS 1.2 Cycle and CCHS 2012 for Mental Health, which contain validated measures for mood and anxiety disorders. ${ }^{34}$ Symptoms will be based on the following questions that have been used in previous studies ${ }^{45-59-64}$ :

- Self-perceived mental health: "In general, would you say your mental health is excellent, very good, good, fair or poor?" ('fair' or 'poor'). This question has been previously validated. ${ }^{65}$ 
- Mood disorder (diagnosed by a health professional, expected to last or already have lasted $\geq 6$ months): "Do you have a mood disorder such as depression, bipolar disorder, mania or dysthymia?” ('yes')

- Anxiety disorder (diagnosed by a health professional, expected to last or already have lasted $\geq 6$ months): "Do you have an anxiety disorder such as a phobia, obsessive-compulsive disorder or a panic disorder?" ('yes')

\section{Precision estimation}

We will compute $95 \%$ CI around the healthcare utilisation rate for each healthcare type (eg, physician visits, spinal imaging). The healthcare utilisation rate will be calculated as the total number of visits for each healthcare type divided by total person-years follow-up in approximately $26000(130000 * 0.2)$ adults with LBP. Assuming a median follow-up of 5 years, eligible respondents will contribute $130000(26000 * 5)$ person-years follow-up. With the least frequent healthcare use being spinal imaging (age/ sex-adjusted annual rate of 4190 per 100000$),{ }^{46} 47$ we estimate that eligible adults will receive 5447 imaging procedures over the study period $(26000 * 5 * 0.0419)$. The estimated overall rate is 0.042 imaging procedures per person-year (95\% CI 0.041 to 0.043 ), based on alpha level of 0.05 . For objective 2 , assuming the most complex fully adjusted model to have 12 covariates ( $\sim 30$ degrees of freedom), $\sim 130000$ subjects ( 26000 having LBP) are assumed adequate for model convergence.

\section{ANALYSIS}

\section{Descriptive analysis}

We will describe the cohort with respect to age, sex, income and other factors related to healthcare use and costs over time. We will use means (SD) or medians (interquartile ranges) and percentages to report continuous and dichotomous variables, respectively. We will report frequency of healthcare use and costs over the study period, stratified by covariates (sociodemographic, health-related factors).

\section{Objective 1}

We will use a weighted logistic regression model that includes the aforementioned covariates to estimate a propensity score for the probability of having LBP (vs no LBP). We will create a propensity score-matched cohort, and hard matched on sex, using a nearest-neighbour 1:1-greedy matching algorithm to match participants in the exposed and control groups based on the logit of the propensity score, with a calliper width of 0.2 times the SD. ${ }^{667}$ We will assess the balance of each baseline covariate between matched exposed and control groups using standardised differences, with differences of $<0.1$ suggesting good balance.$^{68}$ We will use Poisson regression to model the association between LBP and rate of healthcare visits to compute rate ratios (RRs) and 95\% CI, stratified by sex, with robust generalised estimating equations or random effect to account for the matched pair design. We are stratifying analyses by sex because healthcare utilisation patterns for LBP, such as frequency and type of visits, likely differ according to sex. ${ }^{69}$ For each subject, the numerator of the rate is the number of healthcare visits over their follow-up period, while the denominator of the rate is the follow-up duration with offset term to account for varying follow-up. Similarly, we will model differences in healthcare costs calculated as comprehensive per-person costs using linear models (log-transformed) ${ }^{70}$

\section{Objective 2}

The aim is to assess the effect of LBP on healthcare use and costs within strata of mental health symptoms as the effect modifier. We will assess effect modification on the multiplicative and additive scales. The multiplicative scale refers to the estimated joint effect on the RR scale. The additive scale will inform whether the effect of LBP is greater in one subpopulation, which may reflect a biological interaction. In a separate model, we will include an interaction term between LBP and mental health symptoms. Specifically, we will use Poisson and log-transformed linear models for modelling rates of healthcare use and costs, respectively. We will adjust for confounders in the models, which will be selected using the model building strategy proposed by Rothman. ${ }^{71}$ We will then assess effect modification on the additive and multiplicative scales with CIs by calculating the relative excess risk due to interaction (RERI) and ratio of RRs, respectively. ${ }^{72} 73$ RERI would equal 0 in the absence of additive interaction, and ratio of RRs would equal 1 in the absence of multiplicative interaction.

For objectives 1 and 2, we will account for potential overdispersion by introducing a robust SE into the Poisson model or use a negative binomial regression model. To assess model fit (model diagnostics), goodness-of-fit tests (deviance and Pearson $\chi^{2}$ tests) will be used. Bootstrap sampling weights for Ontario from Statistics Canada ${ }^{74}$ will be applied using Balanced Repeated Replication for all analyses and variance calculations to account for the complex survey design. We will use a pooled approach to combine CCHS cycles, which increases sample size and statistical power. ${ }^{74}$ We will adjust for CCHS cycle in all models to account for trends over time. All models and analyses will be run for subpopulations, including by sex, socioeconomic status and gender-related variables (eg, marital status, living arrangements, ethnoracial information from CCHS).

\section{Sensitivity analyses}

We will conduct several sensitivity analyses to assess the potential impact of misclassification and residual confounding on study results. First, we will conduct separate analyses to account for incident episodes of LBP using diagnostic information to assess the potential impact of misclassification of the LBP exposure. We will stratify individuals self-reporting LBP on CCHS by: (1) those with no LBP-related visits in the past 2 years from CCHS interview 
date; (2) those with 1-2 LBP-related visits in the past 2 years; and (3) $>2$ LBP-related visits in the past 2 years. We will use a 2-year look-back window for any physician visits, ED visits, hospitalisations or spinal imaging related to LBP to assess whether associations of interest differed when incorporating LBP diagnostic information. We will conduct separate analyses for each of the three groups to stratify results. Second, we will conduct sub-analyses using the CCHS Cycle 1.2 and CCHS 2012 for Mental Health to assess the validity of self-reported mental health symptoms and the potential impact of misclassification on study results. ${ }^{34}$ Third, to assess the potential impact of residual confounding from unmeasured or unknown confounders, we will conduct a quantitative bias analysis. This analysis aims to estimate the extent to which these confounding variables may explain some or all of the reported association between the exposure and outcome. $^{75}$

Finally, we will conduct sensitivity analyses whereby outcomes are: (1) all-cause healthcare utilisation; (2) cause-specific healthcare utilisation for mental health symptoms and (3) opioid use to inform the generalisability of results. All-cause and mental health-related healthcare utilisation includes physician visits, ED visits and hospitalisations. Mental health-related utilisation will be based on diagnostic codes for mental health disorders, including substance-related disorders, schizophrenia, mood disorders and anxiety disorders (table 2). Opioid use will be assessed as an outcome in a subset of the population due to data availability. Specifically, we will assess opioid use in adults using the Narcotic Monitoring System data from 2012 and onwards, and among those aged $\geq 65$ years or on social assistance using Ontario Drug Benefits data.

\section{Patients and public involvement}

We did not involve patients or the public in the development of this protocol, and they will not be involved in the conduct of the study. We will disseminate study results to patients and the public using website postings, research briefs and social media (table 3 ).

\section{DISCUSSION}

\section{Strengths and limitations}

There are strengths to our study. We will conduct the first population-based study to assess the joint effects of LBP and mental health symptoms on healthcare utilisation and costs, using linked health surveys with health administrative data to identify Ontarians with LBP. Linking population health surveys with health administrative databases is a unique opportunity to identify priority groups of high healthcare use among adults with LBP. Administrative data lack clear approaches to identify individuals with LBP; however, linked population health surveys offer a unique opportunity to build a provincially representative cohort of individuals with LBP. A data linkage approach will ensure capture of all healthcare encounters (medical visits, hospitalisations) in the single
Table 3 Knowledge translation and exchange strategy

\begin{tabular}{|c|c|}
\hline Audience & End-of-grant KTE strategy \\
\hline $\begin{array}{l}\text { Scientific community } \\
\text { (eg, researchers, } \\
\text { academics) }\end{array}$ & $\begin{array}{l}\text { Present results at scientific } \\
\text { conferences in epidemiology and } \\
\text { spine research (eg, Canadian } \\
\text { Society for Epidemiology } \\
\text { and Biostatistics, Society } \\
\text { of Epidemiologic Research, } \\
\text { EUROSPINE) } \\
\text { Submit manuscripts to relevant } \\
\text { high-impact, peer-reviewed } \\
\text { journals (eg, Spine Journal) for } \\
\text { open access publication }\end{array}$ \\
\hline $\begin{array}{l}\text { Researchers, knowledge } \\
\text { users }\end{array}$ & $\begin{array}{l}\text { Draft 1-page research briefs to } \\
\text { be circulated to: (1) Knowledge } \\
\text { Translation Canada (reaches } \\
>2000 \text { researchers and } \\
\text { knowledge users in Canada) } \\
\text { and (2) the Strategy for Patient- } \\
\text { Oriented Research (SPOR) } \\
\text { Evidence Alliance (reaches }>250 \\
\text { researchers and knowledge users } \\
\text { in Canada and abroad) }\end{array}$ \\
\hline $\begin{array}{l}\text { Healthcare professionals, } \\
\text { researchers, general } \\
\text { public }\end{array}$ & $\begin{array}{l}\text { Draft 1-page research briefs to be } \\
\text { posted on Knowledge Translation } \\
\text { Program website at St. Michael's } \\
\text { Hospital, and circulated to Health } \\
\text { Quality Ontario, low back pain } \\
\text { models of care (Inter-professional } \\
\text { Spine Assessment and Education } \\
\text { Clinics, Primary Care Low Back } \\
\text { Pain Pilot), Choosing Wisely } \\
\text { Canada }\end{array}$ \\
\hline $\begin{array}{l}\text { General public, } \\
\text { community }\end{array}$ & $\begin{array}{l}\text { Post key messages through } \\
\text { Twitter campaign }\end{array}$ \\
\hline
\end{tabular}

KTE, knowledge translation and exchange.

payer system of Ontario. In addition, the costing methodology will use observed person-level healthcare cost data to generate total healthcare spending. Overall, findings will help identify and tailor strategies to subgroups with high healthcare utilisation and costs. Identifying priority groups for LBP is crucial to inform decisions to improve population health, healthcare sustainability and quality of care in Canada and beyond.

There are some limitations that we acknowledge:

- CCHS and RPDB data can only be linked for those who agreed to linkage and with valid health card numbers $(>80 \%)$. Excluded persons may differ (eg, may be sicker, with more severe LBP or mental health symptoms) from those included in the cohort, but this risk of bias is low since refusal rates are low. To assess the impact of any potential selection bias, we will conduct a detailed analysis of those who did not participate as we have baseline information from the unlinked participants from the CCHS.

- Since CCHS captures self-reported data (eg, LBP, mental health symptoms), measurement error may 
arise due to social desirability bias or problems with recall. With administrative data, using LBP diagnostic codes may be prone to under-reporting or misclassification. We have identified several ways to overcome this limitation. First, we will not rely solely on self-reported LBP but compare this information with prior healthcare utilisation with back pain diagnostic codes (table 2). Self-report of LBP may capture individuals who do not formally seek healthcare. However, the data do not allow us to identify individuals who develop LBP during the follow-up period or treat LBP as a time-varying exposure, which may lead to misclassification. We understand that both data sources on their own may imperfectly capture those with LBP and there is no existing registry; thus, we are using both data sources to provide a comprehensive approach and represent the best secondary data methods to capture LBP exposure in the general population. Second, individuals with mental health symptoms may under-report the severity of their symptoms. CCHS uses a validated question for self-perceived mental health, and subanalyses will use the CCHS Cycle 1.2 and CCHS 2012 for Mental Health for validation. ${ }^{3465}$

- Healthcare utilisation will exclude services not covered by OHIP, including prescription drugs outside of hospital settings, allied health services (eg, physiotherapy, chiropractic care) and assistive devices (other than for Ontarians aged $\geq 65$ with specific medical conditions or on government assistance). Therefore, our study results will underestimate the burden of healthcare utilisation for LBP. To address this, we will conduct additional analyses using: (1) previous literature on allied health services utilisation for $\mathrm{LBP}^{13} 7677$; (2) self-reported use of physiotherapy and chiropractic services obtained through CCHS and (3) OHIP data prior to the delisting of certain allied health services (eg, physiotherapy, chiropractic care) in 2004 to estimate the proportion of healthcare utilisation and costs likely underestimated in our results.

- We have limited data on opioid use but will evaluate this in a subpopulation for our sensitivity analysis using Narcotic Monitoring System data for eligible adults 2012 and onwards, and Ontario Drug Benefits data for those aged $\geq 65$ years. Future work can evaluate this as more years of data become available through the Narcotic Monitoring System.

- There may be residual confounding from unmeasured or unknown confounders. We will conduct a quantitative bias analysis to estimate the extent to which these confounding variables may explain some or all of the reported association between the LBP and healthcare utilisation/ costs. $^{75}$

\section{Dissemination of results}

We will submit study results for publication in peer-reviewed journals and presentation at scientific conferences. We have devised a multifaceted knowledge translation and exchange strategy to disseminate results and engage with stakeholders (table 3 ).

\section{Author affiliations}

${ }^{1}$ Epidemiology Division, Dalla Lana School of Public Health, University of Toronto, Toronto, Ontario, Canada

${ }^{2}$ Centre for Disability Prevention and Rehabilitation, Ontario Tech University and Canadian Memorial Chiropractic College, Oshawa, Ontario, Canada

${ }^{3}$ Faculty of Health Sciences, Ontario Tech University, Oshawa, Ontario, Canada ${ }^{4}$ Institute of Health Policy, Management and Evaluation, University of Toronto, Toronto, Ontario, Canada

${ }^{5}$ Knowledge Translation Program, Li Ka Shing Knowledge Institute of St Michael's Hospital, Toronto, Ontario, Canada

${ }^{6}$ ICES, Toronto, Ontario, Canada

Contributors JJW and LCR conceptualised the study. PC and ACT advised on the methods and subject content. JJW drafted the protocol. All authors reviewed the protocol and provided critical input and revisions that were incorporated in the final draft. All authors approved the final manuscript prior to its submission.

Funding Funding for this study is supported by the Canada Research Chair held by LCR. LCR is funded by a Tier 2 Canada Research Chair in Population Health Analytics. ACT is funded by a Tier 2 Canada Research Chair in Knowledge Synthesis. PC is funded by a Tier 2 Canada Research Chair in Disability Prevention and Rehabilitation. The funders played no role in the development of this protocol.

Competing interests PC reports the following outside the submitted work: grants from Aviva Canada, other from European Spine Society, personal fees from European Spine Society, grants from Ontario Ministry of Finance, grants from Canadian Institutes of Health Research—Canada Research Chair Program, other from North American Spine Society, grants from Ontario Trillium Foundation, grants from French Chiropractic Association, other from International Academy of Independent Medical Evaluators, other from Griffith University—Whiplash Symposium 2017, other from World Federation of Chiropractic, personal fees from Canadian Chiropractic Protective Association. None of the other authors have potential competing interests to disclose.

Patient consent for publication Not required.

Ethics approval Research ethics approval has been obtained from the University of Toronto Research Ethics Board.

Provenance and peer review Not commissioned; externally peer reviewed.

Open access This is an open access article distributed in accordance with the Creative Commons Attribution Non Commercial (CC BY-NC 4.0) license, which permits others to distribute, remix, adapt, build upon this work non-commercially, and license their derivative works on different terms, provided the original work is properly cited, appropriate credit is given, any changes made indicated, and the use is non-commercial. See: http://creativecommons.org/licenses/by-nc/4.0/.

\section{ORCID iD}

Laura C Rosella http://orcid.org/0000-0003-4867-869X

\section{REFERENCES}

1. World Health Organization. Who global disability action plan 2014 2021. Better health for all people with disability, 2015. Available: http://www.who.int/disabilities/policies/actionplan/Disability_action_ plan_faq.pdf?ua $=1$ [Accessed 1 Aug 2017].

2. Cassidy JD, Carroll LJ, Côté P. The Saskatchewan health and back pain survey. The prevalence of low back pain and related disability in Saskatchewan adults. Spine 1998;23:1860-6. Discussion 7.

3. Cassidy JD, Côté P, Carroll LJ, et al. Incidence and course of low back pain episodes in the general population. Spine 2005;30:2817-23.

4. Hincapié CA, Cassidy JD, Côté P, et al. Whiplash injury is more than neck pain: a population-based study of pain localization after traffic injury. J Occup Environ Med 2010;52:434-40.

5. Walker BF. The prevalence of low back pain: a systematic review of the literature from 1966 to 1998. J Spinal Disord 2000;13:205-17.

6. Carey TS, Evans A, Hadler N, et al. Care-seeking among individuals with chronic low back pain. Spine 1995;20:312-7.

7. Carey TS, Evans AT, Hadler NM, et al. Acute severe low back pain. A population-based study of prevalence and care-seeking. Spine 1996;21:339-44. 
8. GBD 2015 Disease and Injury Incidence and Prevalence Collaborators. Global, regional, and national incidence, prevalence, and years lived with disability for 310 diseases and injuries, 19902015: a systematic analysis for the Global Burden of Disease Study 2015. Lancet 2016;388:1545-602.

9. Global Burden of Disease Study 2013 Collaborators. Global, regional, and national incidence, prevalence, and years lived with disability for 301 acute and chronic diseases and injuries in 188 countries, 19902013: a systematic analysis for the Global Burden of Disease Study 2013. Lancet 2015;386:743-800.

10. Hoy D, March L, Brooks P, et al. The global burden of low back pain: estimates from the Global Burden of Disease 2010 study. Ann Rheum Dis 2014;73:968-74.

11. Dieleman JL, Baral R, Birger M, et al. Us spending on personal health care and public health, 1996-2013. JAMA 2016;316:2627-46.

12. Cypress BK. Characteristics of physician visits for back symptoms: a national perspective. Am J Public Health 1983;73:389-95.

13. Côté P, Cassidy JD, Carroll L. The treatment of neck and low back pain: who seeks care? who goes where? Med Care 2001;39:956-67.

14. Health Quality Ontario. Evidence to improve care. Quality standard: low back pain, 2019. Available: https://www.hqontario.ca/Evidenceto-Improve-Care/Quality-Standards/View-all-Quality-Standards/LowBack-Pain/About [Accessed 1 Mar 2019].

15. Stewart WF, Yan X, Boscarino JA, et al. Patterns of health care utilization for low back pain. J Pain Res 2015;8:523-35.

16. Chou $\mathrm{R}$, Shekelle $\mathrm{P}$. Will this patient develop persistent disabling low back pain? JAMA 2010;303:1295-302.

17. Pinheiro MB, Ferreira ML, Refshauge K, et al. Symptoms of depression as a prognostic factor for low back pain: a systematic review. Spine J 2016;16:105-16.

18. Kobus AM, Smith DH, Morasco BJ, et al. Correlates of higher-dose opioid medication use for low back pain in primary care. J Pain 2012;13:1131-8.

19. Rhee Y, Taitel MS, Walker DR, et al. Narcotic drug use among patients with lower back pain in employer health plans: a retrospective analysis of risk factors and health care services. Clin Ther 2007;29(Suppl):2603-12

20. Wertli MM, Eugster R, Held U, et al. Catastrophizing-a prognostic factor for outcome in patients with low back pain: a systematic review. Spine J 2014;14:2639-57.

21. Wertli MM, Rasmussen-Barr E, Weiser S, et al. The role of fear avoidance beliefs as a prognostic factor for outcome in patients with nonspecific low back pain: a systematic review. Spine $J$ 2014;14:816-36.

22. Shmagel A, Foley R, Ibrahim H. Epidemiology of chronic low back pain in US adults: data from the 2009-2010 National Health and Nutrition Examination Survey. Arthritis Care Res 2016;68:1688-94.

23. Hirsch O, Strauch K, Held H, et al. Low back pain patient subgroups in primary care: pain characteristics, psychosocial determinants, and health care utilization. Clin J Pain 2014;30:1023-32.

24. Beales DJ, Smith AJ, O'Sullivan PB, et al. Low back pain and comorbidity clusters at 17 years of age: a cross-sectional examination of health-related quality of life and specific low back pain impacts. J Adolesc Health 2012;50:509-16.

25. Becker $\mathrm{A}$, Held $\mathrm{H}$, Redaelli $\mathrm{M}$, et al. Low back pain in primary care: costs of care and prediction of future health care utilization. Spine 2010;35:1714-20.

26. Green BN, Johnson CD, Haldeman S, et al. A scoping review of biopsychosocial risk factors and co-morbidities for common spinal disorders. PLoS One 2018;13:e0197987.

27. Patten SB, Williams JV, Wang J. Mental disorders in a population sample with musculoskeletal disorders. BMC Musculoskelet Disord 2006;7:37.

28. Ha JY, Kim ES, Kim HJ, et al. Factors associated with depressive symptoms in patients with chronic low back pain. Ann Rehabil Med 2011;35:710-8

29. McGowan J, Sampson M, Salzwedel DM, et al. PRESS Peer Review of Electronic Search Strategies: 2015 guideline statement. J Clin Epidemiol 2016;75:40-6.

30. Statistics Canada. Canada at a glance 2018: population, 2018. Available: https://www150.statcan.gc.ca/n1/pub/12-581-x/2018000/ pop-eng.htm?HPA=1 [Accessed 1 Apr 2019].

31. Ontario Ministry of Health and Long-term Care. Understanding health care in Ontario, 2012. Available: http://www.health.gov.on.ca/en/ ministry/hc system/ [Accessed 1 Apr 2019].

32. Statistics Canada. Canadian Community Health Survey (CCHS)annual component. Available: http://www23.statcan.gc.ca/imdbbmdi/document/3226_D7 T9 V8-eng.htm [Accessed 20 Oct 2018].

33. Statistics Canada. Canadian Community Health Survey 2003: user guide for the public use Microdata file Catalogue No. 82M0013GPE. Ottawa, ON, Canada: Statistics Canada, 2005.
34. Canadian Community Health Survey (CCHS). Cycle 1.2 mental health and well-being: public use microdata file documentation. Available: http://www23.statcan.gc.ca/imdb-bmdi/document/3226_DLI_D1_ T22_V2-eng.pdf [Accessed 1 Nov 2018].

35. Statistics Canada. Canadian Community Health Survey-Mental Health (CCHS), 2012. Available: http://www23.statcan.gc.ca/imdb/ p2SV.pl?Function=getSurvey\&SDDS $=5015$ [Accessed Jul 1, 2019]

36. ICES. Data dictionary: OHIP library, 2019. Available: https:// datadictionary.ices.on.ca/Applications/DataDictionary/Library.aspx? Library=OHIP [Accessed 1 Apr 2019].

37. Ontario Ministry of Community and Social Services. Ontario works, 2018. Available: www.mcss.gov.on.ca/en/mcss/programs/social/ow/ index.aspx [Accessed 15 Mar 2019].

38. Ontario Ministry of Community and Social Services. Ontario disability support program, 2018. Available: www.mcss.gov.on.ca/en/mcss/ programs/social/odsp/index.aspx [Accessed 15 Mar 2019].

39. Wodchis W, Bushmeneva K, Nikitovic M, et al. Guidelines on person level costing using administrative databases in Ontario. Toronto: Health System Performance Research Network, 2013.

40. Roos LL, Wajda A. Record linkage strategies. Part I: estimating information and evaluating approaches. Methods Inf Med 1991;30:117-23

41. Wong JJ, Côté P, Sutton DA, et al. Clinical practice guidelines for the noninvasive management of low back pain: a systematic review by the Ontario Protocol for Traffic Injury Management (OPTIMa) Collaboration. Eur J Pain 2017;21:201-16.

42. Alkherayf F, Agbi C. Cigarette smoking and chronic low back pain in the adult population. Clin Invest Med 2009;32:360-7.

43. Cassidy T, Fortin A, Kaczmer S, et al. Relationship between back pain and urinary incontinence in the Canadian population. Phys Ther 2017;97:449-54.

44. Bielecky A, Chen C, Ibrahim S, et al. The impact of co-morbid mental and physical disorders on presenteeism. Scand J Work Environ Health 2015;41:554-64.

45. Lim K-L, Jacobs P, Klarenbach S. A population-based analysis of healthcare utilization of persons with back disorders: results from the Canadian Community Health Survey 2000-2001. Spine 2006;31:212-8.

46. Iron K, Jaakkimainen L, Rothwell D, et al. Investigation of acute lower back pain in Ontario: are guidelines being followed? Institute for Clinical Evaluative Sciences, 2004. Available: https://www.ices.on.ca/ flip-publication/investigating-of-acute-lower-back-pain/files/assets/ basic-html/index.html\#1 [Accessed 1 Feb 2018].

47. Fine B, Schultz SE, White L, et al. Impact of restricting diagnostic imaging reimbursement for uncomplicated low back pain in Ontario: a population-based interrupted time series analysis. CMAJ Open 2017:5:E760-7.

48. Rosella LC, Fitzpatrick T, Wodchis WP, et al. High-cost health care users in Ontario, Canada: demographic, socio-economic, and health status characteristics. BMC Health Serv Res 2014;14:532.

49. Bilandzic A, Rosella L. The cost of diabetes in Canada over 10 years: applying attributable health care costs to a diabetes incidence prediction model. Health Promot Chronic Dis Prev Can 2017;37:49-53.

50. Rosella LC, Lebenbaum M, Fitzpatrick T, et al. Impact of diabetes on healthcare costs in a population-based cohort: a cost analysis. Diabet Med 2016;33:395-403.

51. Iles RA, Davidson M, Taylor NF. Psychosocial predictors of failure to return to work in non-chronic non-specific low back pain: a systematic review. Occup Environ Med 2008;65:507-17.

52. Steenstra IA, Munhall C, Irvin E, et al. Systematic review of prognostic factors for return to work in workers with sub acute and chronic low back pain. J Occup Rehabil 2017;27:369-81.

53. Austin PC, Walraven Cvan. The mortality risk score and the ADG score: two points-based scoring systems for the Johns Hopkins aggregated diagnosis groups to predict mortality in a general adult population cohort in Ontario, Canada. Med Care 2011;49:940-7.

54. Chen H, Kwong JC, Copes R, et al. Cohort profile: the Ontario Population Health and Environment Cohort (ONPHEC). Int $J$ Epidemiol 2016.

55. Tu K, Chen Z, Lipscombe LL, et al. Prevalence and incidence of hypertension from 1995 to 2005: a population-based study. CMAJ 2008;178:1429-35

56. Gershon AS, Wang C, Guan J, et al. Identifying individuals with physician diagnosed COPD in health administrative databases. COPD 2009;6:388-94.

57. Hux JE, Ivis F, Flintoft V, et al. Diabetes in Ontario: determination of prevalence and incidence using a validated administrative data algorithm. Diabetes Care 2002;25:512-6.

58. Schultz SE, Rothwell DM, Chen Z, et al. Identifying cases of congestive heart failure from administrative data: a validation 
study using primary care patient records. Chronic Dis Inj Can 2013;33:160-6.

59. Badley EM, Millstone DB, Perruccio AV. Back pain and co-occurring conditions: findings from a nationally representative sample. Spine 2018;43:E935-41.

60. Chiu M, Amartey A, Wang X, et al. Ethnic differences in mental health status and service utilization: a population-based study in Ontario, Canada. Can J Psychiatry 2018;63:481-91.

61. Hategekimana C, Karamouzian M. Self-Perceived mental health status and uptake of fecal occult blood test for colorectal cancer screening in Canada: a cross-sectional study. Int $J$ Health Policy Manag 2016;5:365-71.

62. Lange S, Quere M, Shield K, et al. Alcohol use and self-perceived mental health status among pregnant and breastfeeding women in Canada: a secondary data analysis. BJOG 2016;123:900-9.

63. Davison KM, Holloway C, Gondara L, et al. Independent associations and effect modification between lifetime substance use and recent mood disorder diagnosis with household food insecurity. PLoS One 2018;13:e0191072.

64. Romain AJ, Marleau J, Baillot A. Impact of obesity and mood disorders on physical comorbidities, psychological well-being, health behaviours and use of health services. $J$ Affect Disord 2018;225:381-8.

65. Mawani FN, Gilmour H. Validation of self-rated mental health. Ottawa, ON: Statistics Canada, 2010.

66. Austin PC. Optimal caliper widths for propensity-score matching when estimating differences in means and differences in proportions in observational studies. Pharm Stat 2011;10:150-61.
67. Austin PC, Small DS. The use of bootstrapping when using propensity-score matching without replacement: a simulation study. Stat Med 2014;33:4306-19.

68. Austin PC. Balance diagnostics for comparing the distribution of baseline covariates between treatment groups in propensity-score matched samples. Stat Med 2009;28:3083-107.

69. Mortimer M, Ahlberg G. To seek or not to seek? Care-seeking behaviour among people with low-back pain. Scand J Public Health 2003;31:194-203.

70. Khan NA, Quan H, Bugar JM, et al. Association of postoperative complications with hospital costs and length of stay in a tertiary care center. J Gen Intern Med 2006;21:177-80.

71. Rothman KJ. Epidemiology: an introduction, chapter 12. OUP USA, 2012.

72. VanderWeele TJ, Tchetgen Tchetgen EJ. Attributing effects to interactions. Epidemiology 2014;25:711-22.

73. VanderWeele TJ, Vansteelandt S. Invited commentary: some advantages of the relative excess risk due to interaction (RERI) towards better estimators of additive interaction. Am J Epidemiol 2014;179:670-1.

74. Thomas S, Wannell B. Combining cycles of the Canadian Community Health Survey. Health Rep 2009;20:53-8.

75. Lash TL, Fox MP, Fink AK. Applying quantitative bias analysis to epidemiologic data. Springer Science \& Business Media, 2011.

76. Bath B, Lawson J, Ma D, et al. Self-Reported use of family physician, chiropractor and physiotherapy services among adult Canadians with chronic back disorders: an observational study. BMC Health Serv Res 2018;18:970.

77. Côté P, Baldwin ML, Johnson WG. Early patterns of care for occupational back pain. Spine 2005;30:581-7. 